\title{
Actuación Arqueológica en los depósitos mesolíticos de Cueva de la Cocina (Dos Aguas, Valencia): VALORACiÓn PRELIMINAR
}

(1) Grup d'Investigació PREMEDOC. Dpt. Prehistòria, Arqueologia i Ha Antiga. Universitat de València. oreto.garcia@uv.es, Alfredo.Cortell-Nicolau@uv.es

(2) Investigador postdoctoral "Juan de la Cierva". Grup de Recerca GRAMPO. Dpt. Prehistòria. Universitat Autònoma de Barcelona. salvador.pardo@uab.cat

(3) Grup d'Investigació GRAM. Dpt. Prehistòria, Arqueologia i Hª Antiga. Universitat de València. adiez@uv.es

(4) Museu de Prehistòria - S.I.P. Diputació de València. Joaquim.juan@dival.es

(5) Dpt. of Anthropology. The Pennsylvania State University. sbm19@psu.edu

(6) Dpt. Prehistòria, Arqueologia i $\mathrm{H}^{\mathrm{a}}$ Antiga. Universitat de València. mirco.ramacciotti@gmail.com

\section{INTRODUCCIÓN}

En el año 2015 iniciamos una nueva etapa de actuaciones arqueológicas en el yacimiento prehistórico de Cueva de la Cocina (Dos Aguas, Valencia). El objetivo de este nuevo programa de intervenciones ha consistido en recabar información estratigráfica, paleoeconómica, paleoambiental y cultural sobre los depósitos prehistóricos de esta cavidad, que permitan de este modo una lectura más ajustada de las anteriores excavaciones efectuadas por Lluís Pericot (Pericot 1945; Fortea 1973) y Javier Fortea (Fortea et al. 1987) (fig. 1). Desde esa fecha, y hasta el año 2018, se han realizado una serie de sondeos en campañas de campo anuales financiadas por el Museu de Prehistòria de la Diputació de València. Desde la publicación de las primeras noticias sobre el lugar (Pericot 1945), Cueva de la Cocina se convirtió en una importante referencia para la caracterización tecno-económica y cultural de los últimos cazadores y recolectores holocenos en la fachada Mediterránea de la península Ibérica, adscripción que fue contextualizada y detallada por Javier Fortea (1973) en su trabajo sobre los complejos microlaminares y geométricos del Epipaleolítico. La presencia de evidencias de cultura material de cronología neolítica, especialmente algunos fragmentos de cerámica con decoración impresa en un contexto en el que abundaban los materiales líticos mesolíticos, contribuyó al desarrollo de la hipótesis a propósito de la aculturación del sustrato (Fortea 1973).

Entre los objetivos abordados en el marco de los proyectos HAR2012-33111 MESOCOCINA y HAR201568962 EVOLPAST se encuentran la evaluación de las dinámicas socioecológicas a lo largo de la amplia secuencia mesolítica, así como el análisis del proceso de neolitización en un marco de trabajo diseñado para la obtención y estudio de registros arqueológicos precisos. La interpretación de la secuencia de Cueva de la Cocina constituye uno de los ejes de actuación, nutrido tanto a partir de las nuevas investigaciones de campo, como del análisis de las intervenciones realizadas con anterioridad. En este nuevo marco de trabajo se circunscriben los resultados preliminares que presentamos a continuación a partir de la descripción de la intervención efectuada en el sondeo 4. 


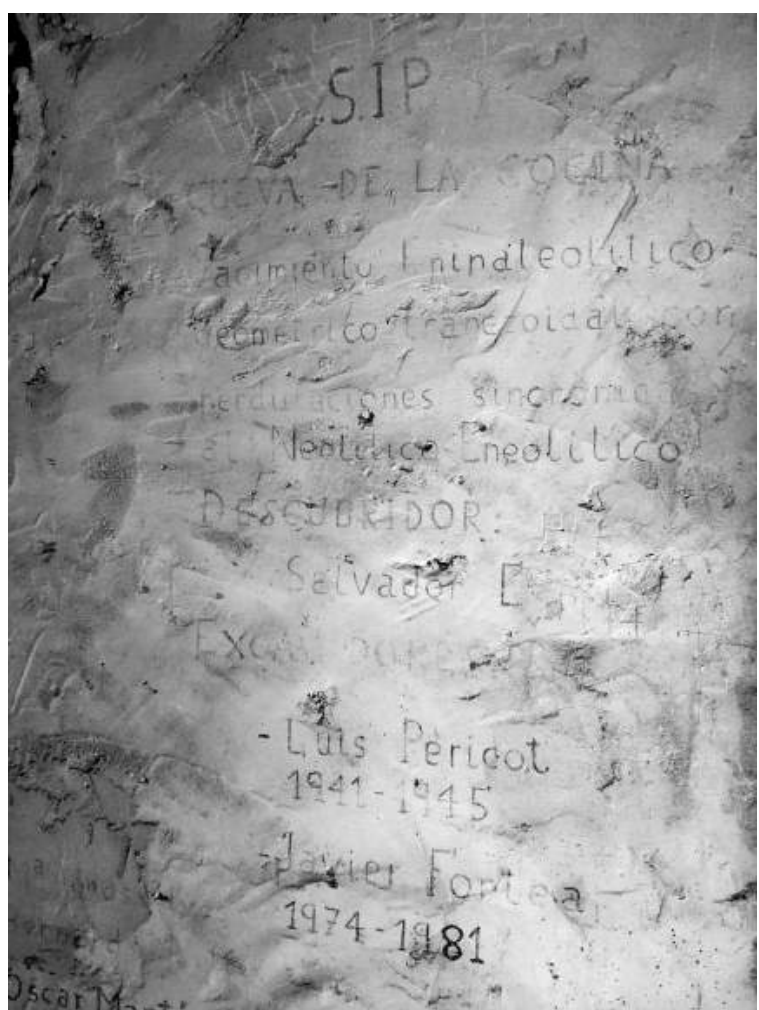

Fig. 1: Muro situado a la entrada de la cavidad (derecha) con indicación de las campañas efectuadas en Cueva de la Cocina y sus participantes.

\section{DESCRIPCIÓN DE LOS TRABAJOS REALIZADOS}

Los trabajos de campo llevados a cabo en esta tercera etapa de actuaciones en la cavidad incluyen a un total de seis sondeos excavados en las inmediaciones de la gran cata efectuada por Lluís Pericot en la entrada, y hacia el interior, en relación con alguna de las intervenciones puntuales de Javier Fortea (García Puchol et al. 2015; 2016). La cueva es amplia y ofrece un espacio bien iluminado en su zona anterior, dónde se concentran las principales evidencias de ocupaciones prehistóricas (fig. 2). Allí se sitúa el área de excavación de los años 1941 y 1945 , cuando se excavaron un total de cuatro grandes catas contiguas (1941, 1942, 1943 y 1945) que alcanzaron el nivel de arcillas de base (Pericot 1945). Esta intervención cuenta con una descripción de los trabajos de campo, acorde con la metodología de la práctica arqueológica en esos momentos, que ha permitido su reconstrucción en base a los datos referidos sobre la extensión y la profundidad de las diferentes capas rebajadas (Diez Castillo et al. 2017).
Por su parte, Javier Fortea planteó una gran cata en la parte intermedia de la cavidad sin conexión con los trabajos de Pericot, así como una zanja que partía del sondeo de Pericot y que comunicaba con un segundo sondeo menor también situado en esta parte intermedia de la cueva. Estas intervenciones fueron recogidas minuciosamente atendiendo a técnicas modernas de excavación, de forma que han podido ser reconstruidas con un gran detalle (Pardo-Gordó et al. 2018).

En este texto nos centraremos en la descripción del sondeo 4 (fig. 3), abierto en la campaña de 2015, sobre el cuál hemos seguido trabajando hasta la última intervención realizada (2018). Se trata de un sondeo de $5,5 \mathrm{~m}^{2} \mathrm{ubi}$ cado en el sector excavado más próximo a la entrada actual de la cavidad, a $1 \mathrm{~m}$ del cerramiento. En la primera campaña pudimos comprobar la conservación de depósitos arqueológicos intactos por debajo de la base del muro antiguo, visible en este tramo al no haberse visto afectado por las excavaciones de Pericot. De este modo pudimos comprobar que por debajo del mismo se desarrollaba un nivel con materiales prehistóricos, aspecto que propició que en las siguientes campañas ampliáramos el sondeo hasta llegar, en el transcurso de la última realizada, al depósito de base prácticamente en toda la extensión rebajada. En el transcurso de la excavación se ha llevado a cabo un registro minucioso de todos los hallazgos y se ha procedido a cribar todo el sedimento con agua. Del mismo modo se ha realizado un registro espacial y fotográfico preciso de las diferentes UUEE que ha permitido la generación de ortofotos y modelos 3D (fig. 3). La descripción que presentamos del depósito excavado así como de las evidencias materiales y biológicas, permiten una primera aproximación a propósito de su interpretación en relación con las ocupaciones mesolíticas en la cavidad.

\section{RESULTADOS}

En un trabajo reciente publicamos el detalle de las fechas radiocarbónicas obtenidas sobre una selección de muestras procedentes de las intervenciones de Pericot y una propuesta de modelización cronológica bayesiana a partir de la información de la secuencia arqueológica y las dataciones (García Puchol et al. 2018). En el año 2018 (Pardo-Gordó et al. 2018) se han dado a conocer las fechas obtenidas sobre una selección de muestras de la intervención efectuada por Fortea (fig. 4). Este nuevo marco permite precisar con mayor detalle la dinámica de 


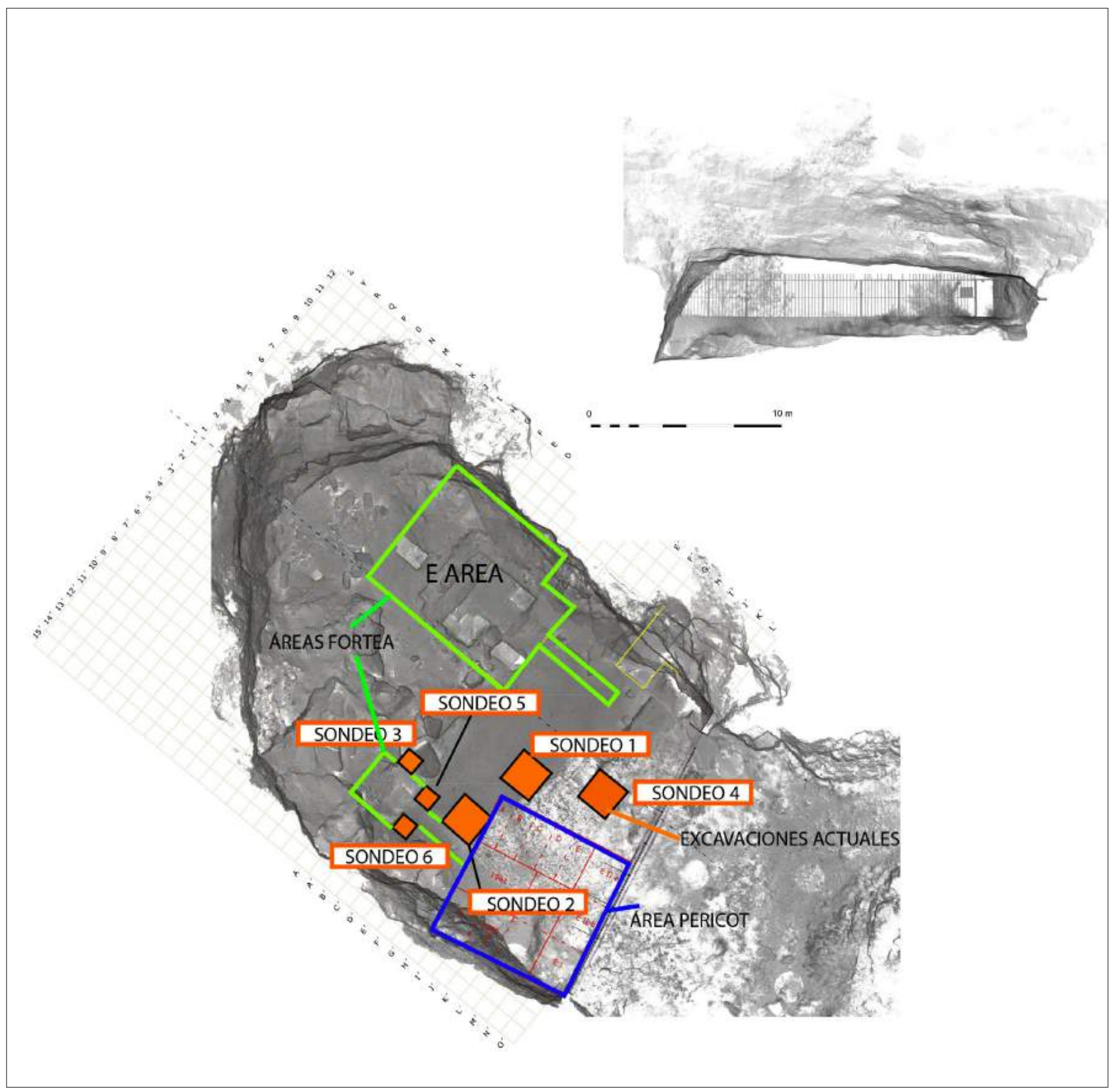

Fig. 2: Plano de la cavidad con la ubicación de los diferentes sectores excavados hasta la actualidad.

las ocupaciones en el yacimiento durante el Mesolítico, y a lo largo del Neolítico y la Edad del Bronce. Las fechas radiocarbónicas corroboran la amplitud de la secuencia mesolítica en la cavidad desde la primera mitad del IX y hasta mediados del VIII milenio cal BP, que incluye el desarrollo de las fases establecidas por Fortea: A (predominio de trapecios) y $\mathrm{B}$ (triángulos tipo Cocina). Una datación del último tercio del VIII milenio cal BP coincide con las evidencias materiales del primer neolítico en la cavidad (presencia de cerámicas impresas cardiales y de gradina). Con los datos actuales se confirmaría la existencia de un hiato entre las últimas ocupaciones del mesolítico y las primeras evidencias de frecuentaciones neolíticas. A este respecto conviene advertir de la desigual preservación de los depósitos neolíticos y posteriores en la cavidad. El tradicional uso de la misma como redil ha propiciado el vaciado periódico de sedimento tal como refieren los testimonios locales, aspecto que ha afectado principalmente a los depósitos más recientes. Otro tipo de procesos naturales han afectado igualmente a los niveles 


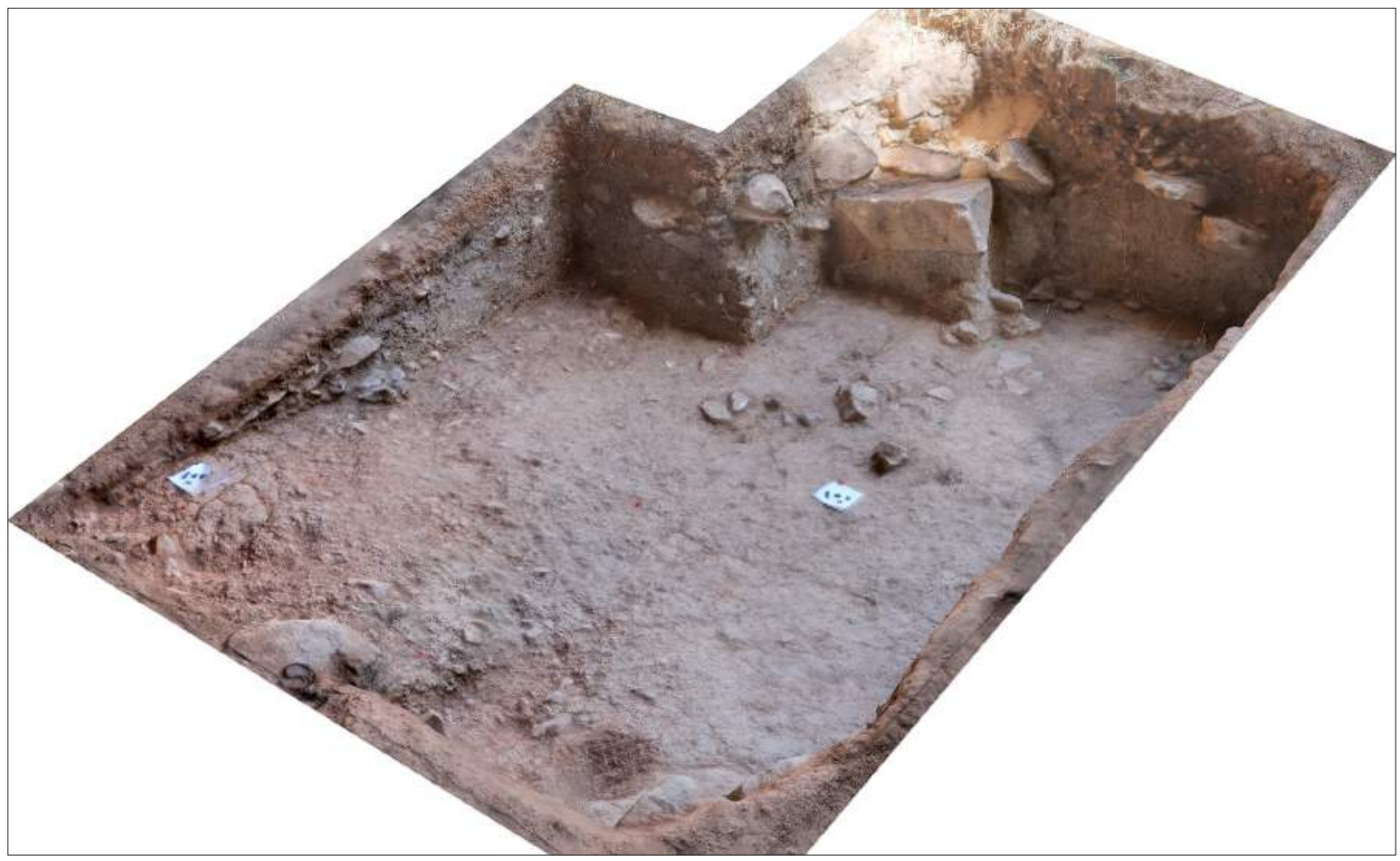

Fig. 3: Modelo 3D del sector 4 al final de la campaña 2018.

arqueológicos, tal como ha podido comprobarse tras el examen de la documentación del sector E excavado por Fortea (Pardo-Gordó et al. 2018). En este caso, los escasos restos de cultura material del Neolítico antiguo (cerámica) aparecen a modo de intrusiones en los depósitos mesolíticos, en clara desconexión con las fechas radiocarbónicas obtenidas. En los sectores excavados por Pericot los fragmentos cerámicos relacionados con ocupaciones neolíticas y posteriores se circunscribieron principalmente al área inmediata a la pared meridional de la cavidad (1942 y 1945 I), principalmente al espacio que se situaba al exterior del muro antiguo de cierre (1945 I y II), ubicación que pudo haber contribuido a su preservación. Este último sector ha sido el único publicado con más detalle hasta la fecha, puesto que a partir del mismo Pericot y Fortea establecieron la secuencia del yacimiento (Fortea 1973).

El sondeo 4 se localiza en el límite entre el antiguo muro de cierre de la cavidad y hacia el exterior del mismo (fig. 2). El hallazgo de su base, descrita también en los diarios de Pericot, permite su ubicación en relación con las catas de 1943 y 1945 II. El depósito prehistórico intacto excavado se corresponde con acumulaciones de ocupaciones de cronología mesolítica. Aunque todavía no disponemos de dataciones relacionadas con este sector, las características de la cultura material y de los restos biológicos recuperados así lo confirmarían. Entre la cultura material abundan los restos de talla y los útiles líticos, con una especial incidencia de las morfologías geométricas triangulares (triángulos tipo Cocina) asociadas a microburiles. Los restos de fauna resultan también numerosos así como la malacofauna, especialmente terrestre, pero también se han hallado restos de origen marino (cerastoderma). Las piezas líticas sobre caliza relacionadas con diferentes actividades de procesado están bien representadas.

El nivel arqueológico fértil es de apariencia homogénea y coloración grisácea, textura arcillosa y con abundantes clastos. Alguna de las concentraciones de piedras detectadas podrían relacionarse con algún tipo de estructura desmantelada, en tanto que en otros casos podría explicarse por aportes naturales dada su proximidad a la boca de entrada. No se distinguen suelos de ocupación, aspecto que será explorado con detalle a partir del análisis espacial de la distribución de los restos en curso de realización. El nivel descansa directamente 


\begin{tabular}{|c|c|c|c|c|c|c|c|c|}
\hline Sondeo/Capa & Muestra & Especie & Ref. laboratorio & ${ }^{14} \mathrm{C}$ age $\mathrm{BP}$ & SD & $8^{13} \mathrm{C}$ & $\delta^{15} \mathrm{~N}$ & $\mathrm{C}: \mathrm{N}$ \\
\hline Pericot 1941 Capa 3 & Hueso & Cervus elaphus & UCIAMS-147346 & 6970 & 35 & $-19,5$ & 4,0 & 3,25 \\
\hline Pericot 1941 Capa 6 & Hueso & Cervus elaphus & UCIAMS-145194 & 7300 & 30 & $-20,3$ & 3,9 & 3,20 \\
\hline Pericot 1941 Capa 8 & Hueso & Capra pyrenaica & UCIAMS-145195 & 7475 & 25 & $-20,4$ & 4,2 & 3,18 \\
\hline Pericot 1941 Capa 11 & Hueso & Capra pyrenaica & UCIAMS-147347 & 7415 & 35 & $-19,5$ & 3,8 & 3,22 \\
\hline Pericot 1941 Capa 13 & Hueso & Capra pyrenaica & UCIAMS-147348 & 7905 & 40 & $-19,0$ & 4,5 & 3,24 \\
\hline Pericot 1945 Capa 6 & Hueso & Capra pyrenaica & Beta-267435 & 6840 & 50 & nd & nd & nd \\
\hline Pericot 1945 Capa 8 & Hueso & Capra pyrenaica & Beta-267436 & 7080 & 50 & nd & nd & nd \\
\hline Pericot 1945 Capa 10 & Hueso & Capra pyrenaica & Beta-267437 & 7050 & 50 & nd & nd & nd \\
\hline Pericot 1945 Capa 12 & Hueso & Capra pyrenaica & Beta-267438 & 7350 & 40 & nd & nd & nd \\
\hline Pericot 1945 Capa 13 & Hueso & Capra pyrenaica & Beta-267439 & 6760 & 40 & nd & nd & nd \\
\hline Pericot 1945 Capa 17 & Hueso & Capra pyrenaica & Beta-267440 & 7610 & 40 & nd & nd & nd \\
\hline 2015 Zanja 3 UE 1030 & Carbón & Rama pimus sp & Beta- 426849 & 6350 & 30 & & & \\
\hline Fortea, D6, Nivel G & Hueso & Ovis aries & UCIAMS-174145 & 4425 & 25 & $-22,4$ & 4,2 & 3,47 \\
\hline Fortea, D5, Nivel H, Capa 4 & Hueso & Ovis aries & UCIAMS-174146 & 3725 & 20 & $-20,3$ & 4,5 & 3,41 \\
\hline Fortea, B5, Nivel H, Capa 2 & Hueso & Capra pyrenaica & UCIAMS-145198 & 6985 & 25 & $-19,2$ & 4,4 & 3,16 \\
\hline Fortea, B4, Nivel H3, Capa 5 & Hueso & Cervus elaphus & UCIAMS-145196 & 7455 & 25 & $-20,2$ & 4,8 & 3,17 \\
\hline Fortea, B4, Nivel H4, Capa 6 & Hueso & Capra pyrenaica & UCIAMS-145197 & 7710 & 30 & $-19,1$ & 4 & 3,15 \\
\hline
\end{tabular}

Fig. 4: Tabla con el detalle de las dataciones del yacimiento publicadas hasta la fecha.

sobre las arcillas pleistocenas que conforman la base de las acumulaciones antrópicas (Fumanal 1986). Su profundidad es variable, adaptándose a la cubeta conformada por el depósito basal, y alcanzado los $40 \mathrm{~cm}$ en el corte meridional. Gran parte del relleno arqueológico de la cavidad se concentra sobre esta amplia depresión situada en la entrada, dónde su máxima cota es de 4,10 m en el sector excavado junto a la entrada y pegado a la pared S (1945 I). Hacia el interior de la cueva su altura disminuía progresivamente hasta alcanzar los límites de la depresión. La situación del sector 4 coincide con uno de los bordes laterales de esta depresión sobre la que se conformó gran parte del depósito arqueológico. Su excavación ha permitido entrever al menos una parte de la historia del desmantelamiento de parte de los niveles arqueológicos superiores en Cueva de la Cocina.

Con los datos actuales sabemos que los niveles superiores de la secuencia, sobre todo aquellos relativos a las ocupaciones del Neolítico y la Edad del Bronce, desaparecieron en gran parte del espacio ubicado en el interior del muro de cierre antiguo, preservándose únicamente en la parte intermedia, donde las grandes losas caídas del techo posiblemente en época moderna (Fumanal 1986) facilitaron su conservación, y en la zona pegada a la pared y al exterior del muro. La propia construcción del mismo descansaba directamente sobre niveles de cronología mesolítica en el sector 4. La excavación de Pericot ofrece pistas también sobre este aspecto puesto que, en los sectores excavados más al interior (1941 y 1943A y B), el nivel de arcillas rojas afloraba en las primeras capas de excavación bajo un relleno que incluía exclusivamente materiales de cronología mesolítica. La extracción masiva de estiércol que tuvo lugar en el periodo transcurrido entre las intervenciones de Pericot y Fortea, descrita en los informes de campo de Fortea, pudo haber agudizado el desmantelamiento en esta área.

A modo de conclusión añadiremos que los trabajos realizados recientemente en el sector 4 han permitido la excavación de depósitos intactos de cronología mesolítica en la cavidad. Su análisis pormenorizado, junto a su contextualización en el marco del conjunto de las intervenciones realizadas, permitirá avanzar en la interpretación de esta interesante secuencia que incluye depósitos del Mesolítico, el Neolítico y la Edad del Bronce.

\section{AGRADECIMIENTOS}

El trabajo presentado se ha llevado a cabo en el marco del proyecto HAR2015-68962-P (MINECO/FEDER): "EVOLPAST: Dinámicas evolutivas y patrones de variabilidad cultural de los últimos cazarecolectores y el primer neolítico en el este peninsular (circa 70004500 cal BC)", Ministerio de Economía y Competitividad, Gobierno de España. Las actuaciones de campo han sido financiadas por el programa de excavaciones SIP/Museu de Prehistòria de la Diputació de València. 
Oreto García Puchol, Salvador Pardo-Gordó, Agustín Diez Castillo, Alfredo Cortell Nicolau, Joaquim Juan Cabanilles, Sarah B. McClure, Mirco Ramacciotti

\section{BIBLIOGRAFÍA}

DIEZ CASTILLO, A.; CORTELL, A.; GARCÍA PUCHOL, O.; ESCRIBÁ, P. (2017): Entorno 3D para el análisis y la recreación virtual de las actuaciones arqueológicas en Cueva de la Cocina (Dos Aguas, Valencia), Virtual Archaeology Review 8, 75-83.

DOI: https://doi.org/10.4995/var.2017.7028

FORTEA, J. (1973): Los complejos microlaminares y geométricos del Epipaleolítico mediterráneo español, Memorias del Seminario de Prehistoria y Arqueología 4, Salamanca.

FUMANAL, M. P. (1986): Sedimentología y clima del País Valenciano: las cuevas habitadas en el Cuaternario reciente, Serie Trabajos Varios. S.I.P. 83, Valencia.

GARCÍA PUCHOL, O.; DIEZ CASTILLO, A.; McCLURE, S. B.; JUAN CABANILLES, J.; PARDO-GORDÓ, S.; CORTELL, A.; ESCRIBÁ, P. (2016): Cueva de la Cocina (Dos Aguas, Valencia): Intervención, metodología, resultados, Saguntum-PLAV 48, 191-195. DOI: https://doi.org/10.7203/SAGVNTVM.48.9203

GARCÍA PUCHOL, O.; JUAN CABANILLES, J.; McCLURE, S. B.; DIEZ CASTILLO, A.; PARDO-GORDÓ, S. (2015): Avance de resultados de los nuevos trabajos arqueológicos en Cueva de la Cocina (Dos Aguas, Valencia): campaña de 2015, Saguntum-PLAV 47, 251-255.

DOI: https://doi.org/10.7203/SAGVNTVM.47.7195

GARCÍA PUCHOL, O.; McCLURE, S. B.; JUAN CABANILLES, J.; DIEZ CASTILLO, A.; BERNABEU, J.; MARTÍ, B.; PARDO-GORDÓ, S.; PASCUAL. J. LL.; PÉREZ, M.; MOLINA, LL.; KENNETT, D. .J. (2018): Cocina cave revisited: Bayesian radiocarbon chronology for the last huntergatherers and first farmers in Eastern Iberia, Quaternary International 472b, 259-271.

DOI: https://doi.org/10.1016/j.quaint.2016.10.037.

PARDO-GORDÓ, S.; GARCÍA PUCHOL, O.; DIEZ CASTILLO, A.; McCLURE, S.; B., JUAN CABANILLES, J.; PÉREZ, M.; MOLINA, LL.; BERNABEU, J.; PASCUAL, J. LL.; KENNETT, D.J.; CORTELL , A.; BASILE, M. (2018): Taphonomic processes inconsistent with indigenous Mesolithic acculturation during the transition to the Neolithic in the Western Mediterranean, Quaternary International 483, 136-147. DOI: https://doi.org/10.1016/j.quaint.2018.05.008

PERICOT, L. (1945): La Cueva de la Cocina (Dos Aguas). Nota preliminar, APL II, 39-71. 